\title{
A Class of Estimator for Population Mean Under SRS
}

\author{
Syed Abdul Rehman ${ }^{* 1}$ and Muhammad Asif ${ }^{2}$ \\ ${ }^{1}$ Department of Statistics, Balochistan University of Information Technology, \\ Engineering and Management Sciences, Balochistan, Pakistan \\ ${ }^{2}$ Department of Statistics, Lasbela University of Agriculture, Water \& Marine Sciences, \\ Balochistan, Pakistan \\ *Corresponding Author: arehman@stat.qau.edu.pk
}

\begin{abstract}
In this paper we propose a class of estimators for the estimation of finite population mean using the auxiliary information when SRS scheme is used. The expressions for the Bias and mean square error (MSE) of the existing and suggested class of estimators are derived up to first degree of approximation and the efficiency comparison of suggested class of estimators is made with other existing estimators, using both theoretically and numerically based on real population sets.
\end{abstract}

Key words: Auxiliary variable, Bias, Mean square error, relative efficiency

\section{Notations and Symbols}

Let we have a population of fixed size $\mathrm{N}$ such that $U=\left\{U_{1}, U_{2}, \ldots \mathbf{K}, U_{N}\right\}$. Each $U_{i}=\left(Y_{i}, X_{i}\right), i=1,2,3, \ldots . ., N$, has a pair of values. Here $\mathrm{Y}$ is the variable under study which is correlated with the auxiliary variable $\mathrm{X}$. let $y=\left\{y_{1}, y_{2}, \ldots \mathrm{K}, y_{n}\right\}$ and $x=\left\{x_{1}, x_{2}, \ldots \mathrm{K}, x_{n}\right\}$ be $\mathrm{n}$ sample selected by using simple random sampling without replacement (SRSWOR), then consider the following notations

$$
\begin{array}{ll}
\bar{Y}=\frac{1}{N} \sum_{i=1}^{N} Y_{i} & \text { Population mean of the studyvariable } \mathrm{Y} \\
\bar{X}=\frac{1}{N} \sum_{i=1}^{N} X_{i} & \text { Population meanof theauxiliaryvariable } \mathrm{X} \\
S_{y}^{2}=\frac{1}{N-1} \sum_{i=1}^{N}\left(Y_{i}-\bar{Y}\right)^{2} & \text { Populationvariance of the StudyvariableY }
\end{array}
$$


$S_{x}^{2}=\frac{1}{N-1} \sum_{i=1}^{N}\left(X_{i}-\bar{X}\right)^{2} \quad$ Populationvariance of the auxiliary variable $\mathrm{X}$ $S_{y x}=\frac{1}{N-1} \sum_{i=1}^{N}\left(Y_{i}-\bar{Y}\right)\left(X_{i}-\bar{X}\right) \quad$ Population covariance among $\mathrm{Y}$ and $\mathrm{X}$ $C_{y}=\frac{S_{y}}{\bar{Y}} \quad$ Population $\mathrm{CV}$ of the study variable $\mathrm{Y}$ $C_{x}=\frac{S_{x}}{\bar{X}} \quad$ Population $\mathrm{CV}$ of the auxiliary variable $\mathrm{X}$ $\rho=\frac{C_{y x}}{C_{y} C_{x}} \quad$ Population Correlation coefficient between $\mathrm{Y}$ and $\mathrm{X}$ $\beta_{1}=\frac{\mu_{3}^{2}}{\mu_{2}^{3}} \quad$ Coefficient of skewness of theauxiliaryvariable $\mathrm{X}$ $\beta_{2}=\frac{\mu_{4}}{\mu_{2}^{2}} \quad$ Coefficient of kurtosis of theauxiliaryvariable $\mathrm{X}$

$\lambda=\left(\frac{1}{n}-\frac{1}{N}\right)$

FPCF

The following useful error terms are considered to derive the expressions for the Bias and MSE of the estimators

$e_{0}=\frac{\bar{y}-\bar{Y}}{\bar{Y}} \quad e_{1}=\frac{\bar{x}-\bar{X}}{\bar{X}} \quad$ Such that $\quad \bar{y}=\bar{Y}\left(1+e_{0}\right)$ and $\quad \bar{x}=\bar{X}\left(1+e_{1}\right)$

The expected values of the above error terms are given as

$$
\begin{array}{ll}
E\left(e_{0}\right)=0 & E\left(e_{1}\right)=0 \\
E\left(e_{0}^{2}\right)=\lambda C_{y}^{2} & E\left(e_{1}^{2}\right)=\lambda C_{x}^{2} \quad E\left(e_{0} e_{1}\right)=\lambda \rho C_{y} C_{x}
\end{array}
$$

Where $\rho$ is the coefficientof correlationbetween study variableY and theauxiliary variable $\mathrm{X}$.

\section{Existing Estimators}

Here we will discourse a number of existing estimators of the population mean available in literature. The expressions for the bias and MSE of each of these estimators up to the first order of approximation are also given 
The usual mean estimator of the study variable is

$t_{n}=\bar{y}=\frac{1}{n} \sum_{i=1}^{n} y_{i}$

The variance of $t_{n}$ is given by

$\operatorname{Var}\left(t_{n}\right)=\lambda \bar{Y}^{2} C_{y}^{2}$

The ratio estimator is given by

$t_{r}=\bar{y}\left(\frac{\bar{X}}{\bar{x}}\right)$

The bias and MSE of $t_{r}$ are given by

$\operatorname{Bias}\left(t_{1}\right)=\lambda \bar{Y}^{2}\left(C_{y}^{2}-\rho C_{y} C_{x}\right)$

and $\operatorname{MSE}\left(t_{1}\right)=\lambda \bar{Y}^{2}\left(C_{y}^{2}+C_{x}^{2}-2 \rho C_{y} C_{x}\right)$

In case of negatively correlation, the product estimator is given by

$t_{p}=\bar{y}\left(\frac{\bar{x}}{\bar{X}}\right)$

Bias and MSE of $t_{p}$ are given by

$\operatorname{Bias}\left(t_{p}\right)=\lambda \bar{Y} \rho C_{y} C_{x}$

and $\operatorname{MSE}\left(t_{p}\right)=\lambda \bar{Y}^{2}\left(C_{y}^{2}+C_{x}^{2}+2 \rho C_{y} C_{x}\right)$

Many researchers modified the above estimators by using the further information of auxiliary variable like median, quartiles, coefficient of skewness and kurtosis etc. Al-Omari et al. (2009) proposed the following ratio type estimator, by using the known first quartile of the auxiliary variable

$t_{q 1}=\bar{y}\left(\frac{\bar{X}+Q_{1}}{\bar{x}+Q_{1}}\right)$

The bias and MSE of $t_{q 1}$ are given by

$\operatorname{Bias}\left(t_{q 1}\right)=\lambda \bar{Y}\left(C_{x}^{2} \theta_{1}^{2}-\rho \theta_{1} C_{y} C_{x}\right)$ 
and $\operatorname{MSE}\left(t_{q 1}\right)=\lambda \bar{Y}^{2}\left(C_{y}^{2}+C_{x}^{2} \theta_{1}^{2}-\rho \theta_{1} C_{y} C_{x}\right)$

where

$$
\theta_{1}=\frac{\bar{X}}{\bar{X}+Q_{1}}
$$

Bahl and Tuteja (1991) proposed the following ratio type estimator

$t_{\text {exp }}=\bar{y} \exp \left(\frac{\bar{X}-\bar{x}}{\bar{X}+\bar{x}}\right)$

Bias and MSE of $t_{\mathrm{exp}}$ are given by

$\operatorname{Bias}\left(t_{\text {exp }}\right)=\lambda \bar{Y}\left(\frac{3}{8} C_{y}^{2}-\frac{1}{2} \rho C_{y} C_{x}\right)$

and $\operatorname{MSE}\left(t_{\mathrm{exp}}\right)=\lambda \bar{Y}^{2}\left(C_{y}^{2}+\frac{1}{4} C_{x}^{2}-\rho C_{y} C_{x}\right)$

Bahl and Tuteja (1991) proposed the following product type estimator

$$
t_{\text {exp } 2}=\bar{y} \exp \left(\frac{\bar{x}-\bar{X}}{\bar{x}+\bar{X}}\right)
$$

Bias and MSE of ${ }^{t_{\exp 2}}$ are given by

$\operatorname{Bias}\left(t_{\exp 2}\right)=\lambda \bar{Y}\left(-\frac{1}{8} C_{x}^{2}+\frac{1}{2} \rho C_{y} C_{x}\right)$

and $\quad M S E\left(t_{\exp 2}\right)=\lambda \bar{Y}^{2}\left(C_{y}^{2}+\frac{1}{4} C_{x}^{2}+\rho C_{y} C_{x}\right)$

By combining ratio and product estimator, Singh and Espejo (2003) proposed the following estimator

$$
t_{r p}=\bar{y}\left[\alpha_{1}\left(\frac{\bar{X}}{\bar{x}}\right)+\left(1-\alpha_{1}\right)\left(\frac{\bar{x}}{\bar{X}}\right)\right]
$$

Where $\alpha_{1}$ is a constant with the optimum value as given by

$$
\alpha_{1_{o p t}}=\frac{1}{2}+\frac{1}{2} \frac{\rho C_{y}}{C_{x}}
$$


Bias and MSE of $t_{r p}$ are given as

$\operatorname{Bias}\left(t_{r p}\right)=\lambda \bar{Y}\left(\left(\frac{1}{2}+\frac{1}{2} \rho \frac{C_{y}}{C_{x}}\right) C_{x}^{2}-\rho^{2} C_{y}^{2}\right)$

and $\operatorname{MSE}\left(t_{r p}\right)=\lambda \bar{Y}^{2} C_{y}^{2}\left(1-\rho^{2}\right)$

Which is equivalent to the min MSE of the linear regression estimator

Tailor and Sharma (2009) reformed the Singh and Espejo (2003) estimator by using the known C.V and coefficient of kurtosis of the auxiliary variable as

$$
t_{t s}=\bar{y}\left[\alpha_{2}\left(\frac{C_{x} \bar{X}+\beta_{2}}{C_{x} \bar{x}+\beta_{2}}\right)+\left(1-\alpha_{2}\right)\left(\frac{C_{x} \bar{x}+\beta_{2}}{C_{x} \bar{X}+\beta_{2}}\right)\right]
$$

where $\alpha_{2}$ is constant with the following optimum value

$\alpha_{2_{\text {opt }}}=\frac{1}{2} \frac{\rho C_{y}}{\theta_{2} C_{x}}$

Bias and MSE of $t_{t s}$ are given by

$\operatorname{Bias}\left(t_{t s}\right)=-\lambda \bar{Y} \rho C_{y}\left(\rho C_{y}-\frac{3}{2} \theta_{2} C_{x}^{2}\right)$

$\operatorname{MSE}\left(t_{t s}\right)=\lambda \bar{Y}^{2} C_{y}^{2}\left[\left(1-\rho^{2}\right) C_{y}^{2}-C_{x}^{2} \theta_{2}^{2}\right]$

where

$\theta_{2}=\frac{\bar{X} C_{x}}{\bar{X} C_{x}+\beta_{2}}$

The min MSE of $t_{t s}$ for the optimum value of $\alpha_{2}$ is equivalent to the min MSE of linear regression estimator i.e.

$\operatorname{Min} \operatorname{MSE}\left(t_{t s}\right)=\lambda \bar{Y}^{2} C_{y}^{2}\left(1-\rho^{2}\right)$

Kumar (2015) used the known median of auxiliary variable and reformed Singh and Espejo (2003) estimator as

$t_{k}=\bar{y}\left[\alpha_{3}\left(\frac{\bar{X}+M d}{\bar{x}+M d}\right)+\left(1-\alpha_{3}\right)\left(\frac{\bar{x}+M d}{\bar{X}+M d}\right)\right]$ 
where $\alpha_{3}$ is constant with the following optimum value

$\alpha_{3_{\text {opt }}}=\frac{1}{2} \frac{\rho C_{y}}{\theta_{3} C_{x}}$

Bias and MSE of $\mathrm{t}_{\mathrm{k}}$, are as below

$\operatorname{Bias}\left(t_{k}\right)=-\lambda \bar{Y} \rho C_{y}\left(\rho C_{y}-\frac{3}{2} \theta_{3} C_{x}^{2}\right)$

$\operatorname{MSE}\left(t_{k}\right)=\lambda \bar{Y}^{2} C_{y}^{2}\left[\left(1-\rho^{2}\right) C_{y}^{2}-C_{x}^{2} \theta_{3}^{2}\right]$

where

$\theta_{3}=\frac{\bar{X}}{\bar{X}+M d}$

The min MSE of $t_{k}$ for the optimum value of $\alpha_{3}$ is equivalent to the min MSE of linear regression estimator i.e

$\operatorname{Min} \operatorname{MSE}\left(t_{k}\right)=\lambda \bar{Y}^{2} C_{y}^{2}\left(1-\rho^{2}\right)$

The very known linear regression estimator is

$t_{\text {reg }}=\bar{y}+b_{y x}(\bar{X}-\bar{x})$

Where $b_{y x}=\frac{s_{y x}}{s_{x}^{2}}$ is coefficient of sample regression. The bias and MSE of $t_{r e g}$ are given by

$$
\operatorname{Bias}\left(t_{r e g}\right)=-\lambda \beta S_{x}\left(\frac{\lambda_{12}}{\rho}-\lambda_{03}\right)
$$

$\min \operatorname{MSE}\left(t_{r e g}\right)=\lambda \bar{Y}^{2} C_{y}^{2}\left(1-\rho^{2}\right)$

Singh et al. (2008) suggested a ratio-cum-product exponential type estimator as

$t_{s}=\bar{y}\left[\alpha_{4} \exp \left(\frac{\bar{X}-\bar{x}}{\bar{X}+\bar{x}}\right)+\left(1-\alpha_{4}\right) \exp \left(\frac{\bar{x}-\bar{X}}{\bar{x}+\bar{X}}\right)\right]$

Where $\alpha_{4}$ constant whose optimum value is given by 
$\alpha_{4_{\text {opt }}}=\frac{1}{2}+\frac{\rho C_{y}}{C_{x}}$

The bias and MSE of $t_{s}$ are given by

$\operatorname{Bias}\left(t_{s}\right)=-\frac{1}{8} \lambda \bar{Y}\left(8 \rho^{2} C_{y}^{2}-4 \rho C_{y} C_{x}-C_{x}^{2}\right)$

$\operatorname{Min} \operatorname{MSE}\left(t_{s}\right)=\lambda \bar{Y}^{2} C_{y}^{2}\left(1-\rho^{2}\right)$

Which is equivalent to the min MSE of linear Regression estimator

\section{Proposed Class of Estimators}

Our proposed class of estimators is in the line of Searls (1964). Here we propose the following class of estimators

$$
\begin{aligned}
& t_{p 1}=k_{1} \bar{y}\left[\alpha_{5}\left(\frac{\bar{X}}{\bar{x}}\right)+\left(1-\alpha_{5}\right)\left(\frac{\bar{x}}{\bar{X}}\right)\right] \\
& t_{p 2}=k_{2} \bar{y}\left[\alpha_{6} \exp \left(\frac{\bar{X}-\bar{x}}{\bar{X}+\bar{x}}\right)+\left(1-\alpha_{6}\right) \exp \left(\frac{\bar{x}-\bar{X}}{\bar{x}+\bar{X}}\right)\right]
\end{aligned}
$$

where $k_{1}, k_{2}, \alpha_{5}$ and $\alpha_{6}$ are constants

Now solving equation (23)

$$
\begin{aligned}
& t_{p 1}=k_{1} \bar{Y}\left(1+e_{0}\right)\left[\alpha_{5}\left(1+e_{1}\right)^{-1}+\left(1-\alpha_{5}\right)\left(1+e_{1}\right)\right] \\
& t_{p 1}=k_{1} \bar{Y}\left[1+e_{0}+\left(1-2 \alpha_{5}\right)\left(e_{1}+e_{0} e_{1}\right)+\alpha_{5} e_{1}^{2}\right] \\
& t_{p 1}-\bar{Y}=\bar{Y}\left[\left(k_{1}-1\right)+k_{1}\left\{e_{0}+\left(1-2 \alpha_{5}\right)\left(e_{1}+e_{0} e_{1}\right)+\alpha_{5} e_{1}^{2}\right\}\right]
\end{aligned}
$$

Bias and MSE of $t_{p 1}$ are given by

$$
\operatorname{Bias}\left(\mathrm{t}_{p 1}\right)=\bar{Y} \mathrm{E}\left[\left(\mathrm{k}_{1}-1\right)+\lambda \mathrm{k}_{1}\left\{\left(1-2 \alpha_{5}\right) \rho C_{y} C_{x}+\alpha_{5} \mathrm{C}_{x}^{2}\right\}\right]
$$

and

$$
\begin{gathered}
\operatorname{MSE}\left(t_{p 1}\right)=\bar{Y}^{2}\left(k_{1}-1\right)^{2}+\lambda \bar{Y}^{2} k_{1}^{2}\left\{C_{y}^{2}+\left(1-2 \alpha_{5}\right)^{2} C_{x}^{2}+2\left(1-2 \alpha_{5}\right) \rho C_{y} C_{x}\right\}+2 \lambda \bar{Y}^{2} k_{1}\left(k_{1}-1\right) \\
\left\{\alpha_{5} C_{x}^{2}+\left(1-2 \alpha_{5}\right) \rho C_{y} C_{x}\right\}
\end{gathered}
$$


Now minimizing (26) w.r.t $\alpha_{5}$ and $k_{1}$, up to zero, we get the following two solution set

Solution set I

$k_{1}=0$ and $\quad \alpha_{5}=\frac{\lambda \rho C_{y} C_{x}+1}{\lambda C_{x}\left(2 \rho C_{y}-C_{x}\right)}$

Solution set II

$k_{1}=\frac{8 \lambda \rho^{2} C_{y}^{2}-6 \lambda \rho C_{y} C_{x}-\lambda C_{x}^{2}-4}{16 \lambda \rho^{2} C_{y}^{2}-8 \lambda \rho C_{y} C_{x}-3 \lambda C_{x}^{2}-4 \lambda C_{y}^{2}-4}$

and $\quad \alpha_{5}=\frac{4 \lambda \rho^{2} C_{x} C_{y}^{2}-3 \lambda \rho C_{y} C_{x}^{2}+2 \lambda \rho C_{y}^{3}-\lambda C_{x}^{3}-\lambda C_{x} C_{y}^{2}-2 \rho C_{y}-2 C_{x}}{C_{x}\left(8 \lambda \rho^{2} C_{y}^{2}-6 \lambda \rho C_{y} C_{x}-\lambda C_{x}^{2}-4\right)}$

by substituting the values of solution set II, we get minimum bias and MSE as

$\operatorname{Bias}\left(t_{p 1}\right)=\frac{\lambda \bar{Y}\left(4 \lambda \rho^{2} C_{x}^{2} C_{y}^{2}-4 \lambda \rho^{2} C_{y}^{4}-2 \lambda \rho C_{y} C_{x}^{3}+4 \lambda \rho C_{x}^{3} C_{x}-\lambda C_{x}^{4}-\lambda C_{x}^{2} C_{y}^{2}-4 \rho^{2} C_{y}^{2}+4 C_{y}^{2}\right)}{4+4 \lambda C_{y}^{2}+3 \lambda C_{x}^{2}+8 \lambda \rho C_{x} C_{y}-16 \lambda \rho^{2} C_{y}^{2}}$

and

$\operatorname{MSE}\left(t_{p 1}\right)=\frac{\lambda \bar{Y}^{2}\left(4 \lambda \rho^{2} C_{x}^{3} C_{y}^{2}-4 \lambda \rho^{2} C_{y}^{4}-2 \lambda \rho C_{y} C_{x}^{3}+4 \lambda \rho C_{y}^{3} C_{x}-\lambda C_{x}^{4}-\lambda C_{x}^{2} C_{y}^{2}-4 \rho^{2} C_{y}^{2}+4 C_{y}^{2}\right)}{4+4 \lambda C_{y}^{2}+3 \lambda C_{x}^{2}+8 \lambda \rho C_{x} C_{y}-16 \lambda \rho^{2} C_{y}^{2}}$

Now solving (24)

$$
\begin{aligned}
& t_{p 2}=k_{2} \bar{y}\left[\alpha_{6} \exp \left\{\frac{-e_{1}}{2}\left(1+\frac{e_{1}}{2}\right)^{-1}\right\}+\left(1-\alpha_{6}\right) \exp \left\{\frac{e_{1}}{2}\left(1+\frac{e_{1}}{2}\right)^{-1}\right\}\right] \\
& t_{p 2}=k_{2} \overline{\mathrm{y}}\left[\alpha_{6} \exp \left\{-\frac{e_{1}}{2}+\frac{e_{1}^{2}}{4}\right\}+\left(1-\alpha_{6}\right) \exp \left\{\frac{e_{1}}{2}-\frac{e_{1}^{2}}{4}\right\}\right] \\
& t_{p 2}=k_{2} \bar{y}\left[\alpha_{6}\left\{1-\frac{e_{1}}{2}+\frac{e_{1}^{2}}{4}+\frac{e_{1}^{2}}{8}\right\}+\left(1-\alpha_{6}\right)\left\{1+\frac{e_{1}}{2}-\frac{e_{1}^{2}}{4}+\frac{e_{1}^{2}}{8}\right\}\right] \\
& t_{p 2}-\bar{Y}=\bar{Y}\left[\left(k_{2}-1\right)+k_{2}\left\{e_{0}+\left(\frac{1}{2}-\alpha_{6}\right)\left(e_{1}+e_{o} e_{1}\right)+\left(\frac{\alpha_{6}}{2}-\frac{1}{8}\right) e_{1}^{2}\right\}\right]
\end{aligned}
$$

Bias and MSE of $t_{p 2}$ are 


$$
\begin{aligned}
\operatorname{Bias}\left(t_{p 2}\right) & =\bar{Y}\left[\left(k_{2}-1\right)+\lambda k_{2}\left\{\left(\frac{1}{2}-\alpha_{6}\right) \rho C_{y} C_{x}+\left(\frac{\alpha_{6}}{2}-\frac{1}{8}\right) C_{x}^{2}\right\}\right] \\
\operatorname{MSE}\left(t_{p 2}\right) & =\bar{Y}^{2}\left(k_{2}-1\right)^{2}+\lambda \bar{Y}^{2} k_{2}^{2}\left[C_{y}^{2}+\left(\frac{1}{2}-\alpha_{6}\right)^{2} C_{x}^{2}+2\left(\frac{1}{2}-\alpha_{6}\right) \rho C_{y} C_{x}\right] \\
+ & 2 \lambda \bar{Y}^{2} k_{2}\left(k_{2}-1\right)\left[\left(\frac{\alpha_{6}}{2}-\frac{1}{8}\right) C_{x}^{2}+\left(\frac{1}{2}-\alpha_{6}\right) \rho C_{y} C_{x}\right]
\end{aligned}
$$

Minimizing (28) w.r.t $\alpha_{6}$ and $k_{2}$, we get the following possible solution sets

Solution set I

$$
k_{2}=0 \text { and } \quad \alpha_{6}=\frac{4 \lambda \rho 2 C_{x} C_{y}-\lambda C_{x}^{2}+8}{4 \lambda C_{x}\left(2 \rho C_{y}-C_{x}\right)}
$$

Solution set II

$$
\begin{gathered}
k_{2}=\frac{1}{8} \frac{16 \lambda \rho^{2} C_{y}^{2}-12 \lambda \rho C_{y} C_{x}+\lambda C_{x}^{2}-8}{4 \lambda \rho^{2} C_{y}^{2}-2 \lambda \rho C_{y} C_{x}-\lambda C_{y}^{2}-1} \\
\alpha_{6}=\frac{2\left(4 \lambda \rho^{2} C_{x} C_{y}^{2}-3 \lambda \rho C_{y} C_{x}^{2}+4 \lambda \rho C_{y}^{3}-2 \lambda C x C_{y}^{2}-4 \rho C_{y}-2 C_{x}\right)}{C_{x}\left(16 \lambda \rho^{2} C_{y}^{2}-12 \lambda \rho C_{y} C_{x}+\lambda C_{x}^{2}-8\right)}
\end{gathered}
$$

By substituting the solution set II, we get the following minimum Bias and MSE

$$
\begin{aligned}
\operatorname{Bias}\left(t_{p 2}\right) & =\frac{\lambda \bar{Y}\left(16 \lambda \rho^{2} C_{y}^{2} C_{x}^{2}+64 \lambda \rho^{2} C_{y}^{4}-16 \lambda \rho C_{y} C_{x}^{3}-\lambda C_{x}^{4}-16 \lambda C_{y}^{2} C_{x}^{2}-192 \rho^{2} C_{y}^{2}+64 C_{y}^{2}\right)}{64\left(4 \lambda \rho^{2} C_{y}^{2}-2 \lambda \rho C_{y} C_{x}-\lambda C_{y}^{2}-1\right)} \\
\operatorname{MSE}\left(t_{p 2}\right) & =\frac{\lambda \bar{Y}^{2}\left(16 \lambda \rho^{2} C_{y}^{2} C_{x}^{2}-64 \lambda \rho^{2} C_{y}^{4}-8 \lambda \rho C_{y} C_{x}^{3}+64 \lambda \rho C_{x} C_{y}^{3}-\lambda C_{x}^{4}-16 \lambda C_{y}^{2} C_{x}^{2}-64 \rho^{2} C_{y}^{2}+64 C_{y}^{2}\right)}{64\left(1-4 \lambda \rho^{2} C_{y}^{2}+2 \lambda \rho C_{y} C_{x}+\lambda C_{y}^{2}\right)}
\end{aligned}
$$

\section{Efficiency Comparison}

Now we will compare the minimum MSE of the proposed class of estimators with other competing estimators discussed above in section 3 and the situations under which proposed estimators performs better than other estimators, are also given.

Condition (i). Comparing Eq. (2) and Eq. (27)

$\operatorname{MSE}\left(t_{p 1}\right)-\operatorname{Var}\left(t_{n}\right)<0$, if 


$$
\frac{\lambda C_{x}^{6}+4 \lambda C_{x}^{4} C_{y}^{2}+4 \lambda C_{x}^{2} C_{y}^{4}+2 \lambda C_{x}^{4} C_{y x}+4 \lambda C_{x}^{2} C_{y}^{2} C_{y x}+4 C_{y x}^{2}}{4 \lambda C_{x}^{2} C_{y x}^{2}+12 \lambda C_{y}^{2} C_{y x}^{2}}>1
$$

Condition (ii). Comparing Eq. (2) and Eq. (29)

$\operatorname{MSE}\left(t_{p 2}\right)-\operatorname{Var}\left(t_{n}\right)<0$, if

$$
\frac{\lambda C_{x}^{6}+16 \lambda C_{x}^{4} C_{y}^{2}+64 \lambda C_{x}^{2} C_{y}^{4}+8 \lambda C_{x}^{4} C_{y x}+64 \lambda C_{x}^{2} C_{y}^{2} C_{y x}+64 C_{y x}^{2}}{16 \lambda C_{x}^{2} C_{y x}^{2}+192 \lambda C_{y}^{2} C_{y x}^{2}}>1
$$

Condition (iii). Comparing Eq. (4) and Eq. (27)

$$
\begin{aligned}
& \operatorname{MSE}\left(t_{p 1}\right)-\operatorname{MSE}\left(t_{r}\right)<0, \text { if } \\
& \qquad \frac{\lambda C_{x}^{6}+2 \lambda C_{x}^{4} C_{y}^{2}+\lambda C_{x}^{2} C_{y}^{4}+\lambda C_{x}^{4} C_{y x}+8 \lambda C_{y x}^{3}+C_{x}^{4}+C_{y x}^{2}}{\lambda C_{y}^{2} C_{x}^{2} C_{y x}+9 \lambda C_{x}^{2} C_{y x}^{2}+3 \lambda C_{y}^{2} C_{y x}^{2}+2 C_{x}^{2} C_{y x}}>1
\end{aligned}
$$

Condition (iv). Comparing Eq. (4) and Eq. (29)

$\operatorname{MSE}\left(t_{p 2}\right)-\operatorname{MSE}\left(t_{r}\right)<0$, if

$$
\frac{\lambda C_{x}^{6}+80 \lambda C_{x}^{4} C_{y}^{2}+64 \lambda C_{x}^{2} C_{y}^{4}+136 \lambda C_{x}^{4} C_{y x}+512 \lambda C_{y x}^{3}+64 C_{x}^{4}+64 C_{y x}^{2}}{64 \lambda C_{y}^{2} C_{x}^{2} C_{y x}+528 \lambda C_{x}^{2} C_{y x}^{2}+192 \lambda C_{y}^{2} C_{y x}^{2}+128 C_{x}^{2} C_{y x}}>1
$$

Condition (v). Comparing Eq. (8) and Eq. (27)

$$
\begin{aligned}
& \operatorname{MSE}\left(t_{p 1}\right)-\operatorname{MSE}\left(t_{r}\right)<0, \text { if } \\
& \frac{\lambda\left(3 \theta_{1}^{2}+1\right) \mathrm{C}_{\mathrm{x}}^{6}+\left[\left(4 \theta_{1} \mathrm{C}_{\mathrm{y}}^{2}+8 \theta_{1} \mathrm{C}_{\mathrm{yx}}+4 \mathrm{C}_{\mathrm{y}}^{2}+2 \mathrm{C}_{\mathrm{yx}}\right) \lambda+4 \theta_{1}\right] \mathrm{C}_{\mathrm{x}}^{4}+4 \lambda \mathrm{C}_{\mathrm{y}}^{2}\left(\mathrm{C}_{\mathrm{y}}^{2}+\mathrm{C}_{\mathrm{yx}}\right) \mathrm{C}_{\mathrm{x}}^{2}+32 \lambda \theta_{1} \mathrm{C}_{\mathrm{yx}}^{3}+4 \mathrm{C}_{\mathrm{yx}}^{2}}{\left.16\left[\left\{\frac{3}{8} \lambda \theta_{1} \mathrm{C}_{\mathrm{x}}^{4}+\left\{\left(\theta_{1}+\frac{1}{2}\right) 2 \mathrm{C}_{\mathrm{yx}}+\frac{1}{2} \theta_{1} \mathrm{C}_{\mathrm{y}}^{2}\right\} \lambda+\frac{1}{2} \theta_{1}\right)\right\} \mathrm{C}_{\mathrm{x}}^{2}+\frac{3}{4} \lambda \mathrm{C}_{\mathrm{y}}^{2} \mathrm{C}_{\mathrm{yx}}\right] \mathrm{C}_{\mathrm{yx}}}
\end{aligned}
$$

Condition (vi). Comparing Eq. (8) and Eq. (29)

$$
\begin{aligned}
& \operatorname{MSE}\left(t_{p 2}\right)-\operatorname{MSE}\left(t_{q 1}\right)<0 \\
& \frac{\lambda C_{x}^{4}+128\left(\theta_{1}^{2}+\frac{1}{16}\right) \lambda \rho C_{y} C_{x}^{3}+\left[\left(64 \theta_{1}^{2}+16\right) \lambda C_{y}^{2}+64 \theta_{1}^{2}\right] C_{x}^{2}+512\left(\theta_{1}^{2}+\frac{1}{8}\right) \lambda \rho C_{x} C_{y}^{3}+64 \lambda C_{y}^{4}+64 \rho^{2} C_{y}^{2}}{256\left[\frac{3}{4} \lambda \rho C_{y}^{3}+\frac{1}{2} \lambda \theta_{1} C_{y}^{2} C_{x}+\lambda \rho C_{x}^{2}\left(\theta_{1}^{2}+\theta_{1}+\frac{1}{16}\right) C_{y}+\frac{1}{2} \theta_{1} C_{x}\right] \rho C_{y}}
\end{aligned}
$$

Condition (vii). Comparing Eq. (10) and Eq. (27)

$\operatorname{MSE}\left(t_{p 1}\right)-\operatorname{MSE}\left(t_{\mathrm{exp}}\right)<0$, if 


$$
\frac{7 \lambda C_{x}^{6}+20 \lambda C_{x}^{4} C_{y}^{2}+16 \lambda C_{x}^{2} C_{y}^{4}+4 \lambda C_{x}^{4} C_{y x}+64 \lambda C_{y x}^{3}+4 C_{x}^{4}+16 C_{y x}^{2}}{64 \lambda C_{x}^{2} C_{y x}^{2}+48 \lambda C_{y}^{2} C_{y x}^{2}+16 C_{x}^{2} C_{y x}}>1
$$

Condition (viii). Comparing Eq. (10) and Eq. (29)

$$
\begin{aligned}
& \operatorname{MSE}\left(t_{p 2}\right)-\operatorname{MSE}\left(t_{\mathrm{exp}}\right)<0 \\
& \qquad \frac{256 \lambda \rho^{3} C_{x} C_{y}^{3}+40 \lambda \rho C_{x}^{3} C_{y}+\lambda C_{x}^{4}+32 \lambda C_{x}^{2} C_{y}^{2}+64 \lambda C_{y}^{4}+64 \rho^{2} C_{y}^{2}+16 C_{x}^{2}}{208 \lambda \rho^{2} C_{x}^{2} C_{y}^{2}+192 \lambda \rho^{2} C_{y}^{4}+64 \lambda \rho C_{x} C_{y}}>1
\end{aligned}
$$

Condition (ix). Comparing Eq. (16) and Eq. (27)

$$
\operatorname{MSE}\left(t_{p 1}\right)-\operatorname{MSE}\left(t_{t s}\right)<0, \text { if }
$$

$$
\frac{\lambda\left[C_{x}^{4}+\left(2 C_{y}^{2}+C_{y x}\right) C_{x}^{2}-4 C_{y x}^{2}\right]^{2}}{\theta_{2}^{2} C_{x}^{4}\left[3 \lambda C_{x}^{4}+C_{x}^{2}\left\{4+\lambda\left(4 C_{y}^{2}+8 C_{y x}\right)\right\}-16 \lambda C_{y x}^{2}\right]}>1
$$

Condition (x). Comparing Eq. (16) and Eq. (29)

$$
\begin{aligned}
& \operatorname{MSE}\left(t_{p 2}\right)-\operatorname{MSE}\left(t_{t s}\right)<0, \text { if } \\
& \frac{\lambda\left[16 \rho^{2} C_{y}^{4}-4 \rho C_{y} C_{x}-C_{x}^{2}-8 C_{y}^{2}\right]^{2}}{\theta_{2}^{2} C_{x}\left[-64+\lambda C_{y}^{2}\left(256 \rho^{2}-64\right)-128 \lambda \rho C_{y} C_{x}\right]}>1
\end{aligned}
$$

Condition (xi). Comparing Eq. (18) and Eq. (27)

$$
\begin{aligned}
& \operatorname{MSE}\left(t_{p 1}\right)-\operatorname{MSE}\left(t_{k}\right)<0, \text { if } \\
& \frac{\lambda\left[C_{x}^{4}+\left(2 C_{y}^{2}+C_{y x}\right) C_{x}^{2}-4 C_{y x}^{2}\right]^{2}}{\theta_{3}^{2} C_{x}^{4}\left[3 \lambda C_{x}^{4}+C_{x}^{2}\left\{4+\lambda\left(4 C_{y}^{2}+8 C_{y x}\right)\right\}-16 \lambda C_{y x}^{2}\right]}>1
\end{aligned}
$$

Condition (xii). Comparing Eq. (18) and Eq. (29)

$$
\begin{aligned}
& \operatorname{MSE}\left(t_{p 2}\right)-\operatorname{MSE}\left(t_{k}\right)<0 \\
& \frac{\lambda\left[16 \rho^{2} C_{y}^{4}-4 \rho C_{y} C_{x}-C_{x}^{2}-8 C_{y}^{2}\right]^{2}}{\theta_{3}^{2} C_{x}\left[-64+\lambda C_{y}^{2}\left(256 \rho^{2}-64\right)-128 \lambda \rho C_{y} C_{x}\right]}>1
\end{aligned}
$$

Condition (xiii). Comparing Eq. (20) and Eq. (27)

$\operatorname{MSE}\left(t_{p 1}\right)-\operatorname{MSE}\left(t_{\text {reg }}\right)<0$ 


$$
\frac{\left(4 \rho^{2} C_{y}^{2}-\rho C_{y} C_{x}-C_{x}^{2}-2 C_{y}^{2}\right)^{2}}{\lambda C_{y}^{2}\left(16 \rho^{2}-4\right)-8 \lambda \rho C_{y} C_{x}-3 \lambda C_{x}^{2}-4}<0
$$

Condition (xiv). Comparing Eq. (20) and Eq. (29)

$$
\begin{aligned}
\operatorname{MSE}\left(t_{p 2}\right)-\operatorname{MSE}\left(t_{\text {reg }}\right)<0 \\
\frac{\left(4 \rho^{2} C_{y}^{2}-\rho C_{y} C_{x}-C_{x}^{2}-2 C_{y}^{2}\right)^{2}}{\lambda C_{y}^{2}\left(16 \rho^{2}-4\right)-8 \lambda \rho C_{y} C_{x}-3 \lambda C_{x}^{2}-4}<0
\end{aligned}
$$

\section{Numerical Study}

Now the theoretical results of the proposed class of estimators are experienced against the existing estimators for the following populations. The percentage relative efficiency (PRE) of the estimators is computed using the succeeding formula

$$
\operatorname{PRE}=\frac{\operatorname{Var}\left(t_{n}\right)}{\operatorname{MSE}\left(t_{i}\right)} \times 100
$$

Population I [Source: Fisher (1936)]

The study variable and auxiliary variable are allocated as;

$Y=$ Petal length in $\mathrm{cm}$ and $X=$ Petal width in $\mathrm{cm}$

The summary statistics of the above data is as follows;

$$
\begin{array}{llrr}
N=150 & n=15 \quad \bar{Y}=1.20 \quad \bar{X}=3.76 \quad C_{y}=0.64 \quad C_{x}=0.47 S_{y x}=1.30 \\
\rho=0.96 & \mathrm{Md}=4.35 \quad \beta_{2}=1.60 \quad Q_{1}=1.60
\end{array}
$$

Population II [Source: AMIS (2014)]

The study variable and auxiliary variable are allocated as;

$Y=$ Production of maze in " 000 " tones and $X=$ Area in " 000 " hectares

The summary statistics of the above data is as follows;

$$
\begin{array}{llllll}
N=120 & n=8 & \bar{Y}=30 & \bar{X}=8 & C_{y}=2.95 & C_{x}=1.97 \\
S_{y x}=1303.32 & \rho=0.85 & M d=0.95 & \beta_{2}=11.58 & Q_{1}=0.0 &
\end{array}
$$

Population III [Source: Rudalf et al. (2006)]

The study variable and auxiliary variable are allocated as;

$Y=$ Total irrigated area (in hectares) and $X=$ Total number of Tractors 
The summary statistics of the above data is as follows;

$$
\begin{gathered}
N=69 \quad \begin{array}{l}
n=8 \\
M d=15
\end{array} \quad S_{y x}=4467.69 \beta_{2}=6.41 \quad Q_{1}=10
\end{gathered} \quad C_{x}=0.23 \rho=0.90
$$

Population IV [Source: Waugh (1995)]

The study variable and auxiliary variable are allocated as;

$Y=$ Whole weight of an abalone and $X=$ Length of an abalone

The summary statistics of the above data is as follows

$$
\begin{array}{lllll}
N=4177 & n=25 & \bar{Y}=0.8287 & \bar{X}=0.5240 & C_{y}=0.5917 C_{x}=0.2292 \\
S_{y x}=0.0545 & \rho=0.93 & M d=0.545 & \beta_{2}=0.063 & Q_{1}=0.45
\end{array}
$$

Population V [Source: Arora (2017)]

The study variable and auxiliary variable are allocated as $Y=$ Atmospheric concentrations of MEI and $X=$ Atmospheric concentrations of $\mathrm{CH} 4$ The summary statistics of the above data is

$$
\begin{aligned}
& N=308 \quad n=20 \quad \bar{Y}=0.276 \quad \bar{X}=1749.83 C_{y}=3.404 C_{x}=0.026 S_{y x}=-4.559 \\
& \rho=-0.11 \quad M d=1764.04 \beta_{2}=2.643 \quad Q_{1}=1722.18
\end{aligned}
$$

Population VI [Source: Arora (2017)]

The study variable and auxiliary variable are allocated as;

$Y=$ The mean stratospheric Aerosol optical depth at $550 \mathrm{~nm}$ and $X=$ Atmospheric concentrations of CFC. 12

The summary statistics of the above data is as follows

$$
\begin{array}{llll}
N=308 & n=20 & \bar{Y}=0.0167 & \bar{X}=497.53 \quad C_{y}=1.744 C_{x}=0.116 S_{y x}=-0.410 \\
\rho=-0.244 & M d=528.36 & \beta_{2}=3.111 & Q_{1}=472.411
\end{array}
$$

Population VII [Source: Arora (2017)]

The study variable and auxiliary variable are allocated as;

$Y=$ The mean stratospheric Aerosol optical depth at $550 \mathrm{~nm}$ and $X=$ Atmospheric concentrations of nitrous oxide $\mathrm{N} 2 \mathrm{O}$

The summary of the statistics is as follows 


$$
\begin{aligned}
& N=308 \quad n=15 \quad \bar{Y}=0.0167 \quad \bar{X}=312.39 C_{y}=1.744 C_{x}=0.017 \rho=-0.244 \\
& M d=311.507 S_{y x}=-0.0536 \beta_{2}=0.145 \quad Q_{1}=308.11
\end{aligned}
$$

Population VIII [Source: Agrestic (2012)]

The study variable and auxiliary variable are allocated as;

$Y=$ Total registered votes in a state 1996 and $X=$ Total votes taken by Ross Perot

The summary statistics of the above data is

$$
\begin{array}{cll}
N=67 \quad n=8 & \bar{Y}=79115.6 & \bar{X}=7220.54 \quad C_{y}=1.49 C_{x}=1.24 \rho=0.94 \\
M d=3739 & S_{y x}=989089058 & \beta_{2}=5.98 Q_{1}=1072.50
\end{array}
$$

Table 1: PRE of the estimators

\begin{tabular}{|l|l|l|l|l|l|l|l|l|}
\hline Estimator & Data1 & Data2 & Data3 & Data4 & Data5 & Data6 & Data7 & Data8 \\
\hline $\mathrm{t}_{\mathrm{n}}$ & 100.00 & 100.00 & 100.00 & 100.00 & 100.00 & 100.00 & 100.00 & 100.00 \\
\hline $\mathrm{t}_{\mathrm{r}}$ & 806.94 & 322.70 & 525.78 & 230.80 & 100.16 & 102.89 & 100.67 & 741.18 \\
\hline $\mathrm{t}_{\mathrm{q} 1}$ & 368.12 & 322.70 & 388.80 & 152.02 & 100.08 & 101.57 & 100.34 & 5986 \\
\hline $\mathrm{t}_{\text {exp }}$ & 234.71 & 184.08 & 267.20 & 147.25 & 100.08 & 101.54 & 100.34 & 254.21 \\
\hline $\mathrm{t}_{\mathrm{ts}}$ & 526.58 & 156.16 & 2025 & 555.75 & 101.13 & 106.20 & 114.28 & 234.22 \\
\hline $\mathrm{t}_{\mathrm{k}}$ & 450.04 & 228.83 & 1509 & 6994 & 101.12 & 105.87 & 114.27 & 121.94 \\
\hline $\mathrm{t}_{\mathrm{reg}}, \mathrm{t}_{\mathrm{s}}, \mathrm{t}_{\mathrm{rp}}$ & 1368.01 & 361.33 & 530.05 & 694.97 & 101.13 & 106.32 & 114.28 & 801.95 \\
\hline $\mathrm{t}_{\mathrm{pl}}$ & 1406.85 & 370.21 & 545.56 & 7161 & 1754 & 127.84 & 132.76 & 802.20 \\
\hline $\mathrm{t}_{\mathrm{p} 2}$ & 1521.76 & 440.51 & 532.22 & 718.69 & 1754 & 127.76 & 132.76 & 989.62 \\
\hline
\end{tabular}

\section{Conclusions}

In this article we have proposed an efficient class of estimators for the estimation of finite population mean under SRS. All the compulsory conditions of the data, for which the proposed class of estimators produce better estimates than usual estimator, ratio, exponential, Al- Omari et al. (2009), Singh and Espejo (2003), Tailor and Sharma (2009), Kumar (2015), Linear regression and Singh et al. (2008) estimator, are derived in section 5. An empirical study based on eight real life populations is also carried out to verify the theoretical results.

From the above study and results shown in Table 1, it is easily evident that our suggested class of estimators at their optimal conditions will always perform better than other competing estimators 
when the efficiency conditions, discussed in section 5 are fulfilled by the data under study. Due to the minimum MSE of the proposed class of estimator, it is strongly recommended to use the proposed class of estimators in practical situations for the precise estimation of population mean.

\section{Acknowledgments}

The author would like to thank the Editor and Manage team for the support and guidance in this research project.

\section{References}

Agrestic, A. (2012). Buchanan vote in 2000. University of Florida. Retrieved from users.stat.ufl.edu/ aa/cda/data.html

AMIS. (2014). District wise data of maize, Punjab, Pakistan. Retrieved from http://www.amis.pk/Agristatistics/DistrictWise/Maize.html

Arora, A. (2017). Climate change data from 1983 to 2008. Retrieved fromhttps://rpubs.com/aashish/ClimateChangeDataAnalysis

Bahl, S. and Tuteja, R. K. (1991). Ratio and product type exponential estimator. Information and Optimization Sciences, 12(2): 353-367.

Cochran, W. G. (1940). The estimation of the yield of the cereal experiments by sampling for the ratio of grain to total produce. The Journal of Agriculture Science, 30: 262-275.

Cochran, W. G. (1997). Sampling Techniques. Third edition, Wiley Eastern Limited.

Dubey, V. and Singh, S. K. (2001). An improved regression estimator for estimating population mean. Journal of the Indian society of Agriculture Statistics, 54(2): 179-18

Fisher. R. A. (1936). Iris plants database. Retrieved fromhttps://archive.ics.uci.edu/ml/machinelearning-databases/iris/iris.data

Jeffery C. S. (1987). 1985 auto Imports database. Retrieved from https://archive.ics.uci.edu/ml/machine-learning-databases/autos/imports-85.data

Kaur. (1985). An efficient regression type estimator in survey sampling. Biometrical Journal, 27(1): 107-110.

Kumar, S. (2015). An estimator of mean estimation of study variable using median of auxiliary variable. Sri Lankan Journal of Applied Statistics, 16(2): 107-115.

Mehta, N. and Mandowara, V. L. (2016). A modified ratio-cum-product estimator of finite population mean using ranked set sampling. Communications in Statistics-Theory and Methods, 45: 267-276. 
Murthy, M. N. (1967). Sampling theory and methods. Statistical Publishing Society Calcutta, India, pg.228

Murthy, M. N. Sankhya, A. (1964). Product method of estimation. The Indian Journal of

Statistics, 26: 69-74.

Omari, A. and Ibrahim, K. (2009). New ratio estimators of the mean using simple random sampling and ranked set sampling methods, Revista Investigacion Operacional, 30: 97108.

Pandy, G. S. (1980). Product-cum-power estimators. Calcutta Statistical Association, 32:47-56.

Prasad, B. (1989). Some improved ratio type estimators of population mean and ratio in finite population sample surveys. Communications in Statistics-Theory and Methods, 18: 379392.

Rao, T. J. (1991). On certain method of improving ratio and regression estimators. Communications in Statistics-Theory and Methods, 20(10): 3325-3340.

Robson, D. S. (1957). Application of multivariate Polykays to the theory of unbiased ratio type estimators. Journal of the American Statistical Association, 52: 511-522.

Rudalf, J. F. William J. W. and Sa, P. (2006). Regression analysis statistical modeling of a response variable; $2^{\text {nd }}$ edition. Academic Press; pg. 186-187 and 242-24

Shabbir, J. and Gupta, S. (2010). On estimating the finite population mean in simple and stratified random sampling. Communications in Statistics-Theory and Methods, 40(2): 199-212.

Shabbir, J. and Gupta. S. (2008). On improvement in estimating the population mean in simple random sampling. Journal of Applied Statistics, 35(2): 559-566.

Singh, H. P. (1986). Estimation of ratio, product and mean using auxiliary information in sample surveys. Aligarh Journal of Statistics, 6: 32-44.

Singh, H. P. and Espejo, M. R. (2003). On linear regression and ratio estimator using coefficient of variation of auxiliary variate. The Statisticians, 52(1): 59-67.

Singh, H. P. Tailor, R. and Kakran, M. S. (2004). An improved estimator of population means using power transformations. Journal of the Indian Society Agriculture Statistics, 58: 223-230.

Singh, M. P. (1967). Ratio-cum-product method of estimation. Metrika, 12: 34-42

Singh, R. and Mangat. N. S. (1996). Elements of Survey Sampling. Springer, Netherland 
Singh, R. Chauhan, P. and Sawan, N. (2008). On linear combination of ratio and product type exponential estimator for estimating the finite population mean. Statistics in Transition, 9(1): 105-115.

Tailor, R. and Sharma. B. (2009). A modified ratio-cum-product product estimator of finite population mean using known coefficient of variation and coefficient of kurtosis. Statistics in Transition, 10: 15-24.

Takahasi, K. and Wakimoto, D. K. (1968). On unbiased estimates of the population mean based on the sample stratified by means of ordering. Annals of the Institute of Statistical Mathematics, 20: 1-31.

Upadhyaya, L. N. and Singh, H. P. (2001). Estimation of population standard deviation using auxiliary information. American Journal of Mathematics and Management Sciences, 21(3-4): 245-358.

Upadhyaya, L. N. and Singh. H. P. (1999). Use of transformed auxiliary variable in estimating the finite population mean. Biometrical Journal, 41(5): 627-636.

Waugh. S. (1995). Abalone data. Retrieved from https://archive.ics.uci.edu/ml/machine-learningdatabases/abalone/abalone.data

Some conversions of Suggested estimator

\begin{tabular}{|l|l|l|}
\hline \multicolumn{1}{|c|}{$\mathrm{k}$} & \multicolumn{1}{|c|}{$\alpha$} & \multicolumn{1}{|c|}{ Estimator } \\
\hline $\mathrm{k}$ & $\alpha$ & $t_{p 1}=k \bar{y}\left[\alpha\left(\frac{\bar{X}}{\bar{x}}\right)+(1-\alpha)\left(\frac{\bar{x}}{\bar{X}}\right)\right]$ Proposed estimator \\
\hline 1 & $\alpha$ & $t_{r p}=\bar{y}\left[\alpha\left(\frac{\bar{X}}{\bar{x}}\right)+(1-\alpha)\left(\frac{\bar{x}}{\bar{X}}\right)\right]$ Singh and Espejo (2003) \\
\hline $\mathrm{k}$ & 1 & $t_{\text {kaur }}=k \bar{y}\left(\frac{\bar{X}}{\bar{x}}\right) \operatorname{Kaur}(1986)$ \\
\hline $\mathrm{k}$ & 0 & $t_{\text {kaur }}=k \bar{y}\left(\frac{\bar{x}}{\bar{X}}\right) \operatorname{Kaur}(1986)$ \\
\hline 1 & 1 & $t_{r}=\bar{y}\left(\frac{\bar{X}}{\bar{x}}\right) \quad \operatorname{Cochran}(1940)$ \\
\hline 1 & 0 & $t_{p}=\bar{y}\left(\frac{\bar{x}}{\bar{X}}\right) \quad$ Robson (1967) \\
\hline
\end{tabular}

Some conversions of suggested estimator 
Rehman, S. A. and Asif, M. (2020)

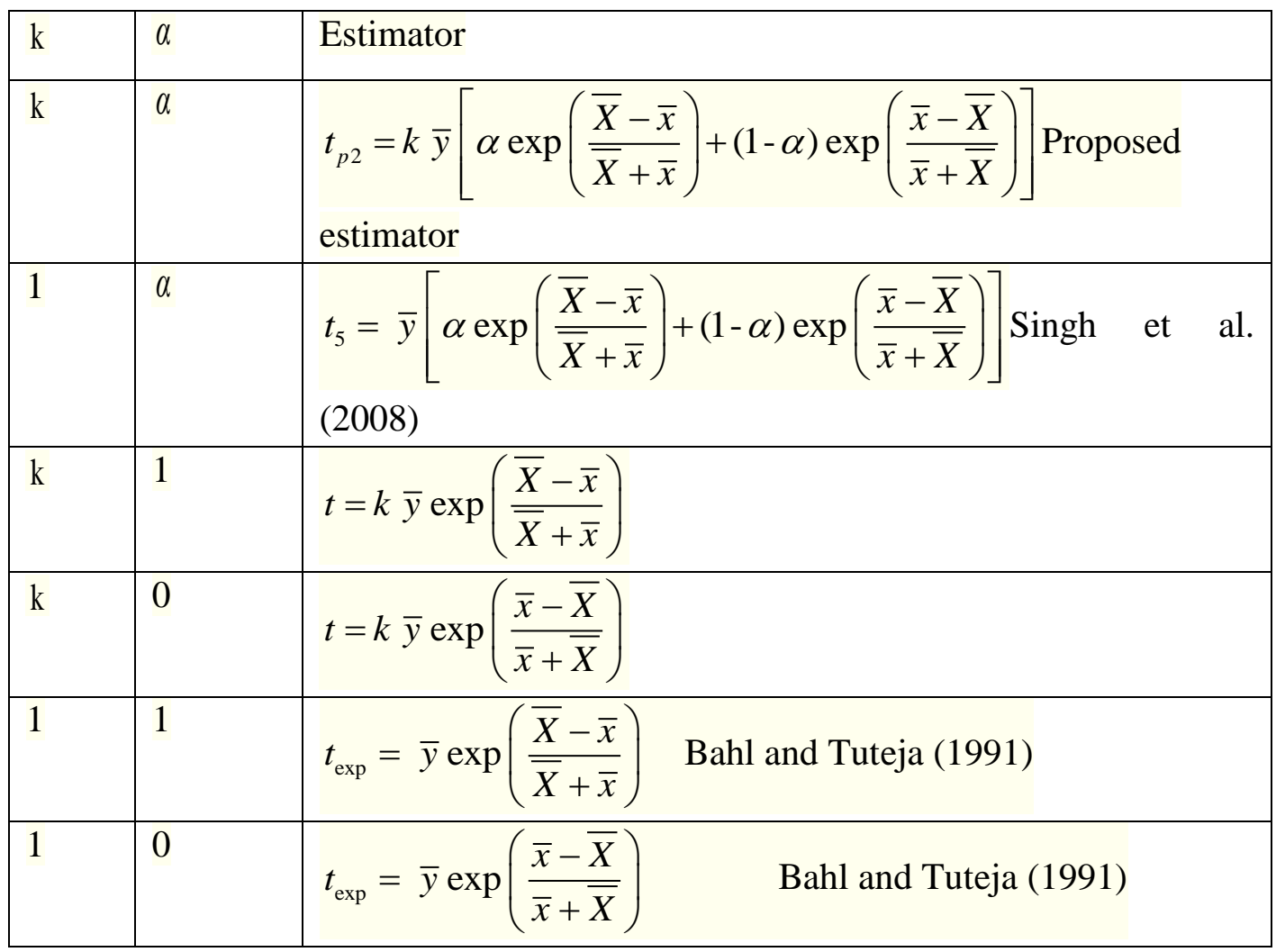

